\title{
Mineração
}

\section{Conversibilidade entre distribuições probabilísticas usadas em modelos de hidrociclones}

\author{
José Aurélio Medeiros da Luz \\ Universidade Federal de Ouro Preto - UFOP \\ Escola de Minas - Departamento de Engenharia de Minas - DEMIN \\ E-mail: jaurelio@demin.ufop.br
}

\section{Resumo}

Apresenta-se uma análise conceitual sobre o relacionamento entre o parâmetro $\boldsymbol{m}$, descritor da agudez da distribuição estatística de Rosin-Rammler, e o parâmetro $\boldsymbol{\alpha}$, seu correspondente na distribuição logística de Whiten. A curva de partição de sólidos, segundo uma função de distribuição de Rosin-Rammler, é adotada no modelo de hidrociclone de Plitt, enquanto que, alternativamente, a função de Whiten é adotada para a partição no modelo de Lynch e Rao. Os resultados mostram que os dois parâmetros podem ser relacionados através da seguinte equação de conversão, obtida por análise de regressão não-linear:

$\alpha=34,555 \times \exp \left[-\frac{3,9}{m^{0,5984}}\right]$

Palavras-chave: probabilidade, distribuição de RosinRammler, distribuição de Whiten, hidrociclones.

\section{Abstract}

This article presents a conceptual analysis on the relationship between the sharpness parameter $\boldsymbol{m}$, of the Rosin-Rammler distribution, and the parameter $\boldsymbol{\alpha}$, its correspondent in the Whiten logistic distribution function. The solids split curve is approximated by a Rosin-Rammler distribution function in the Plitt model for hydrocyclone, while, alternatively, the logistic distribution function is adopted for the partition in the Lynch-Rao model. The results show that the two parameters can be related to the following equation, achieved by nonlinear regression analysis:

$\alpha=34,555 \times \exp \left[-\frac{3,9}{m^{0,5984}}\right]$

Keywords: probability, Rosin-Rammler distribution, Whiten distribution, hydrocyclones. 


\section{Introdução}

Distribuições de probabilidade são utilizadas em várias instâncias da engenharia. A utilização de distribuição probabilística para a previsibilidade da partição de fluxos de particulados em equipamentos de processos, por exemplo, é um procedimento clássico.

A curva de partição de sólidos, segundo uma distribuição de Rosin-Rammler, é adotada no modelo de hidrociclone de Plitt (Plitt, 1976), enquanto que, alternativamente, a distribuição logística modificada (conhecida como função de Whiten) é adotada para a partição no modelo de Lynch e Rao (Lynch, 1987).

Esse artigo apresenta uma análise conceitual sobre o relacionamento entre o parâmetro $\boldsymbol{m}$, descritor da agudez da distribuição estatística de Rosin-Rammler (caso particular da distribuição de Weibull) e o parâmetro $\boldsymbol{\alpha}$, seu correspondente na distribuição logística modificada de Whiten (doravante referida somente como logística, por concisão).

\section{Revisão da literatura}

Modelos matemáticos de classificadores granulométricos, em especial, hidrociclones, têm sido empregados intensivamente na prática industrial. Um desenho esquemático de hidrociclone é mostrado na Figura 1.

Tais modelos baseiam-se, via de regra, na descrição da curva de partição de sólidos, expurgada do bypass (perpasso, ou curto-circuito), isto é, após a subtração do material particulado erroneamente direcionado aos dois fluxos de produtos. Isso porque pode haver o arraste, tanto de finos pela parcela de líquido que se reporta ao underflow, quanto de grossos para o overflow (fluxo de finos, ou "lamas").

Como seria de se esperar, o bypass de grossos para o overflow, é menor que aquele de finos para o underflow, e de modo geral, é considerado desprezível.

Dos dois modelos consagrados, o modelo de Plitt utiliza uma curva de partição expurgada (impropriamente referida como “corrigida”), segundo uma distribuição de Rosin-Rammler, expressa por (Plitt, 1976) (Beraldo, 1987) (Carrisso e Correia, 2002):

$Y_{i c}=1-\exp \left[-\left(\frac{d_{i}}{d^{\prime}}\right)^{m}\right]=1-\exp \left[-\ln (2)\left(\frac{d_{i}}{d_{50 c}}\right)^{m}\right]$

Onde:

- $\boldsymbol{Y}_{\text {ic }}$ - fração do material na classe granulométrica i efetivamente reportado ao fluxo de areias pelo processo classificatório [-].

- $\boldsymbol{d}_{\boldsymbol{i}}$ - tamanho (diâmetro) da classe $\boldsymbol{i}[\mathrm{m}]$.

- $\boldsymbol{d}^{\prime}$ - tamanho característico da distribuição (fator de escala) [m].

- $\boldsymbol{d}_{50 c}$ - tamanho nominal de corte ( $\boldsymbol{d}_{50}$ "corrigido") [m].

- $\boldsymbol{m}$ - parâmetro de agudez (ou módulo da distribuição) [-].
O diâmetro $\boldsymbol{d}^{\prime}$ refere-se ao tamanho no qual 63,21 \% do material se reportam ao underflow (expurgado o curto-circuito). O diâmetro de corte ("corrigido", para o qual $Y_{\text {ic }}=50$ \%), quando expresso em $\mu \mathrm{m}$, pode ser previsto pelas dimensões e operações operacionais do ciclone, no modelo de Plitt por:

$d_{50 c}=\frac{52,45 d_{c}^{0,46} d_{i}^{0,6} d_{o}^{1,21} \exp \left[0,063 c_{v}\right]}{d_{u}^{0,71} h^{0,38} Q_{v}{ }^{0,45} \sqrt{\left(\rho_{s}-\rho_{f}\right)}}$

Onde:

- $\boldsymbol{d}_{\boldsymbol{c}}$ - diâmetro efetivo (interno) do ciclone [cm].

- $\boldsymbol{d}_{\boldsymbol{o}}$ - diâmetro efetivo (interno) do overflow [cm].

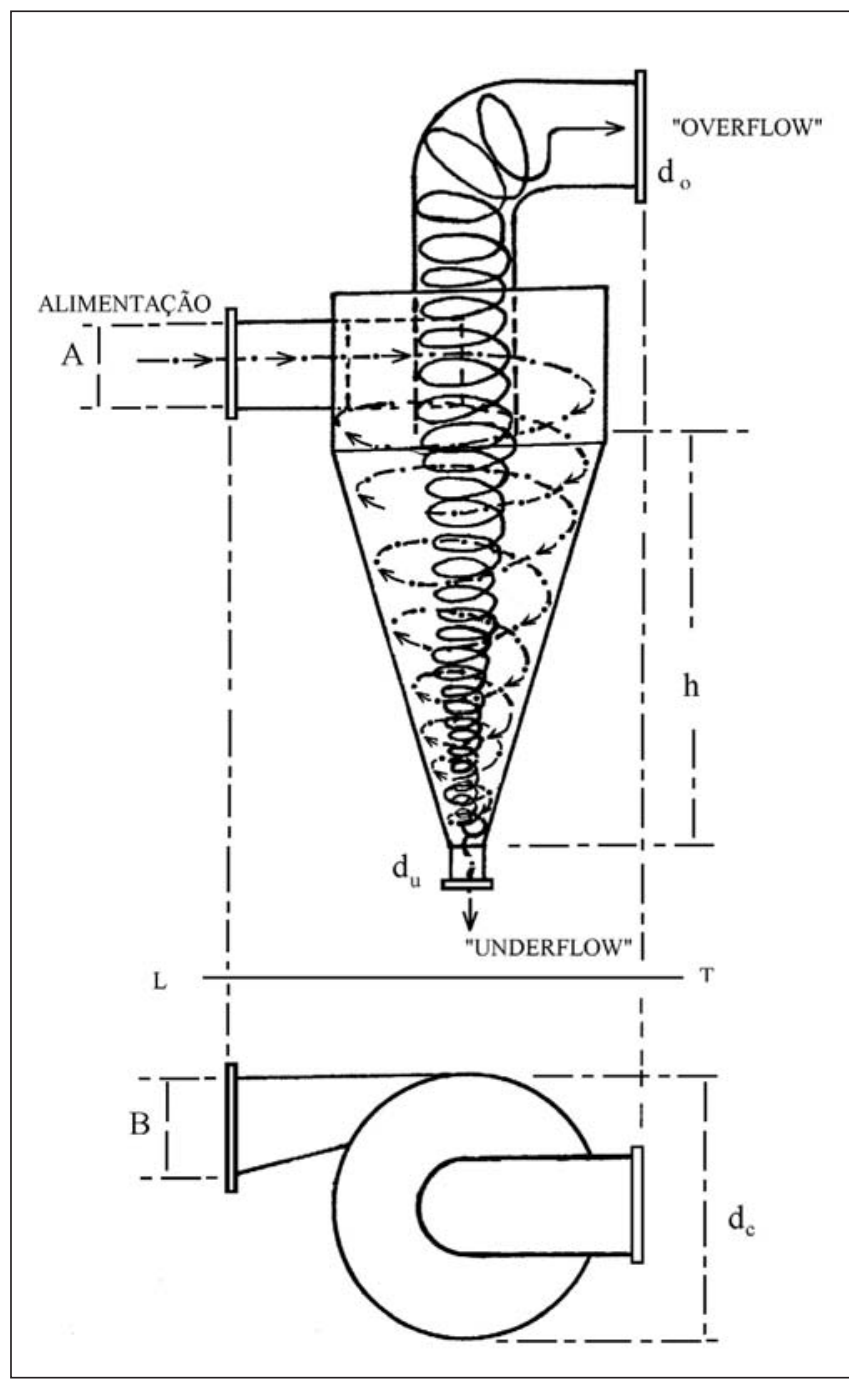

Figura 1 - Desenho esquemático (em épura) de um hidrociclone típico com bocal de injeção retangular, mostrando-se linhas de fluxo hipotéticas. As dimensões consignadas referem-se a valores efetivos (internos). O diâmetro de injeção é o diâmetro com área equivalente à da secção transversal da entrada: $A x B$. A altura livre (h) vai do início do vortex finder (captor de vórtex) até o início do ápex da secção cônica. 
- $\boldsymbol{d}_{u}$ - diâmetro efetivo (interno) do underflow [cm].

- $\boldsymbol{d}_{\boldsymbol{i}}$ - diâmetro equivalente de injeção [cm].

- $c_{v}$ - concentração volumétrica de sólidos na alimentação [\%].

- $\boldsymbol{h}$ - altura livre do ciclone [cm].

- $\boldsymbol{Q}_{v}$ - vazão volumétrica da alimentação [l/min].

- $\boldsymbol{\rho}_{s}$ - massa específica das partículas $\left[\mathrm{t} / \mathrm{m}^{3}\right]$.

- $\boldsymbol{\rho}_{f}$ - massa específica do fluido $\left[\mathrm{t} / \mathrm{m}^{3}\right]$.

O diâmetro de corte real $\left(\boldsymbol{d}_{50}\right)$, por sua vez, leva em conta o bypass. Comumente adota-se que tal curto-circuito, em todas as classes granulométricas, é proporcional à partição de fluido (líquido). Nesse caso, a partição real será expressa por:

$Y_{i}=\left(1-R_{f}\right) \times Y_{i c}+R_{f}$

A expressão para cálculo do diâmetro de corte real $\left(\boldsymbol{d}_{50}\right)$, tendo-se a partição fracional de fluido $\left(\boldsymbol{R}_{f}\right)$ para o underflow, pode ser obtida fazendo $\boldsymbol{Y}_{\boldsymbol{i}}=0$,5 e substituindo $\boldsymbol{Y}_{i c}$ pela equação 1. Assim, um mero algebrismo produz a equação:

$d_{50}=\left[-1,4427 \times \ln \left(\frac{0,5}{1-R_{f}}\right)\right]^{\frac{1}{m}} \times d_{50 c}$

Os valores de $\boldsymbol{R}_{f}$ para hidrociclones em tratamento de minerais ficam tipicamente entre 0,15 e 0,35.

O parâmetro de agudez, $\boldsymbol{m}$, também dito módulo da distribuição, pode ser previsto, segundo Plitt, por:

$m=1,08 \times \exp \left[0,58-1,58 \times\left(\frac{S}{S+1}\right)\right] \times\left[\frac{d_{c}^{2} \times h}{Q_{v}}\right]^{0,15}$

Onde $\boldsymbol{S}$ é a relação entre vazão volumétrica de polpa no underflow e de polpa no overflow, a qual é dada por:

$S=\frac{1,9 \times\left(\frac{d_{u}}{d_{o}}\right)^{3,31} h^{0,54}\left(d_{u}^{2}+d_{0}^{2}\right)^{0,36} \exp \left[0,0054 c_{v}\right]}{H^{0,24} d_{c}^{1,11}}$

Onde o símbolo novo é:

• H - pressão de operação em metro de coluna de polpa [m].

Alternativamente, Lynch e Rao (Lynch \& Rao, 1968; Lynch, 1987) adotaram a seguinte equação logística modificada (função de Whiten) para a equação de partição expurgada ("corrigida"):

$Y_{\text {ic }}=\frac{\exp \left[\alpha\left(\frac{d_{i}}{d_{50 c}}\right)\right]-1}{\exp \left[\alpha\left(\frac{d_{i}}{d_{50 c}}\right)\right]+\exp [\alpha]-2}$
Onde:

- $\alpha$ - parâmetro de agudez da distribuição logística [-].

Plitt (Plitt, 1976) afirma que: "o relacionamento entre $\boldsymbol{\alpha}$ e $\boldsymbol{m}$ pode ser aproximado pela equação:"

$\boldsymbol{\alpha}=1,54 \boldsymbol{m}-0,47$

Em excelente artigo sobre o tema, Nageswararao (Nageswararao, 1999) comenta pesquisas feitas por Asomah na Austrália, que levaram ao desenvolvimento da seguinte correlação empírica entre o parâmetro á e as variáveis dimensionais e operacionais de hidrociclone:

$\alpha=\frac{K_{\text {exp }} \times\left(\frac{d_{o}}{d_{c}}\right)^{1,046} \times \beta^{0,429} \times\left(\frac{\rho_{s}}{\rho_{s}-\rho_{p}}\right)^{2,182}}{d_{c}^{0,148} \times(\operatorname{Re})^{0,107} \times\left(\frac{d_{u}}{d_{c}}\right)^{0,161} \times\left(\frac{\eta_{p}}{\eta_{f}}\right)^{0,854} \times \exp \left[0,094\left(\frac{\theta}{180^{\circ}}\right)\right]}$

Onde os símbolos novos são:

- $K_{\text {exp }}$ - constante experimental do sistema [-].

- Re - número de Reynolds na entrada do hidrociclone [-].

- $\beta$ - ângulo cônico do hidrociclone [ $\left.{ }^{\circ}\right]$.

- $\theta$ - ângulo entre o eixo longitudinal do hidrociclone e a vertical [ $\left.{ }^{\circ}\right]$.

- $\eta_{p}, \eta_{f}$ - viscosidades dinâmicas da polpa e do fluido, respectivamente [Pa.s]

- $\boldsymbol{\rho}_{p}$ - massa específica da polpa (suspensão) $\left[\mathrm{t} / \mathrm{m}^{3}\right]$.

Na equação anterior, as dimensões dos diâmetros devem ser expressas em centímetros. Note-se que as equações anteriores (equações 2, 5, 6 e 8) às vezes são mostradas na literatura com valores discrepantes, atribuíveis a deslizes de edição, os quais, por exemplo, existem no apêndice do artigo original de Plitt, quando as equações são formatadas no sistema métrico (Plitt, 1976).

\section{Metodologia}

O procedimento metodológico usual para um serviço da natureza do presente trabalho foi seguido. O programa computacional de análise estatística de dados gráficos utilizado foi o EasyPlot for Windows, versão 4.0.4, desenvolvido por S. Karon, da Spiral Software (www.spiralsoftware.com).

O principal aspecto da paleta de ajuste estatístico de curvas do programa utilizado é que a essa ferramenta possibilita a adoção arbitrária do tipo de curva. O operador pode selecionar o tipo de curva de ajuste, a partir de uma análise matemática da nuvem de dados, em especial com a introdução de argumentação heurística, baseada no conhecimento do sistema real. Naturalmente, ao menos um dos coeficientes da equação digitada deverá permanecer indeterminado para que o algoritmo de regressão não-linear possa trabalhar. Esse algoritmo usa um filtro de MarquardtLevenberg (o qual, ao contrário do algoritmo de busca por simplex descendente, permite a estimação das incertezas associadas aos valores de regressão). 
Na simulação, funções de distribuições hipotéticas de Rosin-Rammler foram previamente selecionadas (e traçadas no EasyPlot), adotando-se um valor fixo de $\boldsymbol{d}_{50 c}$ (igual a 200 unidades lineares) e variando o valor do expoente (com um passo de 0,1 ), numa faixa que abrangesse a maior parte das aplicações no âmbito do tratamento de minérios, a saber: $0<\boldsymbol{m} \geq 7,0$.

Cada uma dessas curvas de base foi traçada com 200 pares ordenados $\left(\boldsymbol{Y}_{i c} ; \boldsymbol{d}_{i}\right)$. Em seguida, efetuou-se, para cada uma delas, a análise de regressão, selecionando-se uma curva de ajuste seguindo a distribuição logística de Whiten. O programa, então, calculava o valor otimizado para o expoente $\boldsymbol{\alpha}$, a partir da semente inicial injetada pelo operador no algoritmo e seguindo o critério de mínimos quadrados e máxima verossimilhança.

Note-se, entretanto, que (ao menos genericamente falando) esse procedimento de convergência é sensível ao valor da semente inicial, podendo apresentar variações nos valores de regressão e nos valores de incerteza. Desse modo, a análise criteriosa das condições iniciais é de suma relevância nesse tipo de tarefa.

De posse dos pares de valores $\boldsymbol{m}$ e $\boldsymbol{\alpha}$, assim obtidos, procedeu-se à análise de regressão para a obtenção de uma equação que tivesse forte aderência estatística em relação à nuvem de pontos “experimentais".

Equações de regressão diversas, como polinômios, função exponencial e função de Weibull, foram testadas. Naturalmente, como se trata de uma metodologia empírica (em oposição à abordagem “mecanística”) a eleição da função de regressão só se justifica sob o ponto de vista de maior aderência estatística e menores riscos de extrapolações catastróficas (como costuma acontecer com os polinômios). Embora a análise das funções já mostrasse que o valor do diâmetro médio de corte corrigido $\left(\boldsymbol{d}_{50)}\right)$ não influenciaria o resultado, análises suplementares por amostragem aleatória de alguns casos foram feitas para verificação dessa hipótese.

\section{Resultados}

Os resultados da simulação de pontos experimentais e sua correspondente regressão podem ser avaliados na Tabela 1.
A equação seguinte resultou em melhor aderência, para uma faixa mais ampla de $\mathrm{m}$ (entre 0 e 7 ), usando-se os dados da Tabela 1 e o ponto $(0 ; 0)$ :

$$
\alpha=34,555 \times \exp \left[-\frac{3,9}{m^{0,5984}}\right]
$$

A equação anterior revelou excelente precisão, com todos os valores de $\boldsymbol{d}_{50 c}$ testados. O coeficiente de regressão foi de 99,9 \%. A Figura 2, a seguir, ilustra a aderência da equação sugerida à nuvem de dados. Uma regressão polinomial entre $\boldsymbol{m}$ e $\boldsymbol{\alpha}$ (com os mesmos dados), resulta na equação seguinte:

$$
\alpha=0,01216 \times m^{4}-0,18846 \times m^{3}+0,90307 \times m^{2}+0,26272 \times m-0,2383
$$

A equação anterior teve correlação de 99,8 \%, mas superestima valores, em caso de extrapolação. Polinômios de grau 2 e 6, embora com coeficientes de regressão altos, foram menos vantajosos (do segundo grau com menor aderência nos valores de $\boldsymbol{m}$ abaixo de 0,5 e do sexto grau, com aberração entre 5 e 7). Já a equação de Weibull, embora tenha revelado boa aderência no âmbito dos dados, foi preterida pelo seu caráter sigmoidal, o que implicaria, na extrapolação, uma assíntota conceitualmente inexistente.

\section{Conclusão}

Os resultados mostram que os parâmetros de agudez das distribuições de Rosin-Rammler e logística podem ser relacionados, equação 10, com excelente aderência estatística. Provavelmente, os parcos recursos computacionais da

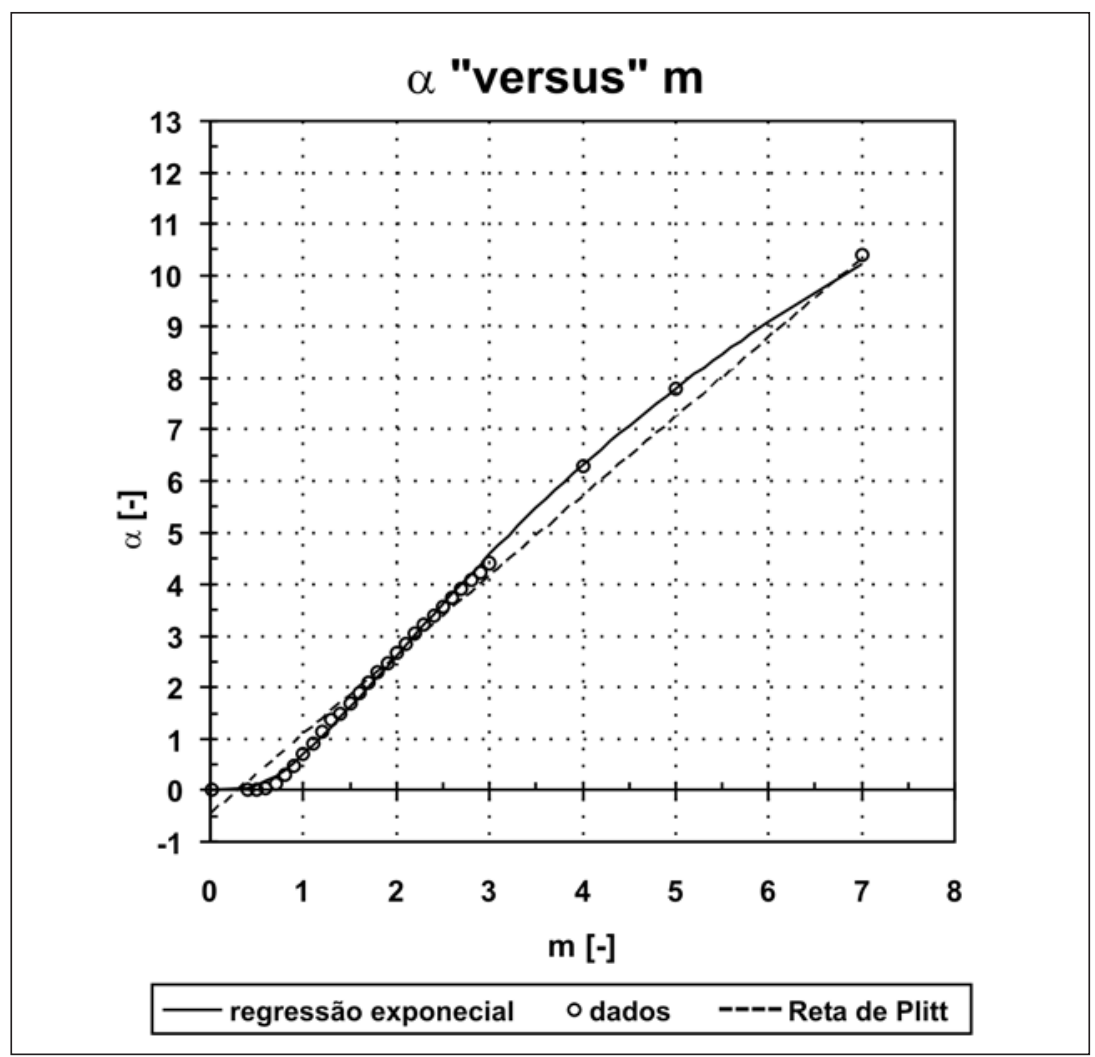

Figura 2 - Regressão (equação 10) para os valores de á da curva de regressão exponencial, em função do parâmetro de agudez da distribuição de Rosin-Rammler e mostrando a reta de Plitt. 
Tabela 1 - Nuvem de pontos de regressão entre $\boldsymbol{m}$ e $\boldsymbol{\alpha}$, mostrando a incerteza (desviopadrão) e coeficiente de regressão percentual (200 pontos por regressão).

\begin{tabular}{|c|c|c|c|}
\hline $\begin{array}{c}m \\
\text { (adotado) }\end{array}$ & $\begin{array}{c}\alpha \\
\text { (por regressão) }\end{array}$ & $\begin{array}{l}\sigma_{\alpha} \\
{[-]}\end{array}$ & $\begin{array}{l}R^{2} \\
{[\%]}\end{array}$ \\
\hline 0,4 & 0,0572 & 2,440 & 88,4 \\
\hline 0,5 & 0,0265 & 0,0138 & 96,7 \\
\hline 0,6 & 0,032 & 0,0149 & 99,6 \\
\hline 0,7 & 0,139 & 0,0144 & 99,9 \\
\hline 0,8 & 0,293 & 0,0107 & 100,0 \\
\hline 0,9 & 0,487 & 0,0059 & 100,0 \\
\hline 1,0 & 0,693 & 0,0000 & 100,0 \\
\hline 1,1 & 0,914 & 0,0045 & 100,0 \\
\hline 1,2 & 1,14 & 0,0088 & 100,0 \\
\hline 1,3 & 1,37 & 0,0128 & 100,0 \\
\hline 1,4 & 1,48 & 0,0248 & 100,0 \\
\hline 1,5 & 1,69 & 0,0091 & 100,0 \\
\hline 1,6 & 1,89 & 0,0106 & 100,0 \\
\hline 1,7 & 2,09 & 0,0119 & 100,0 \\
\hline 1,8 & 2,29 & 0,0130 & 100,0 \\
\hline 1,9 & 2,48 & 0,0139 & 100,0 \\
\hline 2,0 & 2,67 & 0,0149 & 100,0 \\
\hline 2,1 & 2,86 & 0,0154 & 100,0 \\
\hline 2,2 & 3,05 & 0,0167 & 100,0 \\
\hline 2,3 & 3,23 & 0,0181 & 100,0 \\
\hline 2,4 & 3,41 & 0,0193 & 100,0 \\
\hline 2,5 & 3,58 & 0,0206 & 100,0 \\
\hline 2,6 & 3,75 & 0,0222 & 100,0 \\
\hline 2,7 & 3,92 & 0,0233 & 100,0 \\
\hline 2,8 & 4,09 & 0,0247 & 100,0 \\
\hline 2,9 & 4,25 & 0,0263 & 100,0 \\
\hline 3,0 & 4,42 & 0,0260 & 100,0 \\
\hline 4,0 & 6,3 & 0,0103 & 99,99 \\
\hline 5,0 & 7,8 & 0,0111 & 99,93 \\
\hline 7,0 & 10,4 & 0,1290 & 99,92 \\
\hline
\end{tabular}

época de lançamento desses modelos clássicos de ciclone aqui descritos impediram maior acurácia na consolidação da equação (6).

A busca de maior significado conceitual na equação de regressão eleita, embora desejável, seria tarefa inútil, visto que as próprias equações reduzidas adotadas para partições de ciclones (como nos modelos de Plitt e de LynchRao) não têm maior significação conceitual. Simplesmente representam a aderência de pontos, os quais estão atrelados por fenômenos complexos, mas que se podem resumir na seguinte afirmação: "partículas grosseiras têm maior probabilidade de se reportarem ao produto grosseiro". Tais fenômenos usualmente se descrevem por algoritmos de Monte Carlo, até mesmo usando-se distribuição diversa (que não Rosin-Rammler) para a alimentação, como a log-normal (Zhao, 2005). Como observação final, o autor vê como potencialmente proveitoso, à luz dos recursos atuais, revisar antigas abordagens de quantificação dos fenômenos ligados aos aspectos tecnológicos que ainda se mantêm estimulantes.

\section{Referências bibliográficas}

BERALDO, J. L. Moagem de minérios em moinhos tubulares. São Paulo: PróMinério/Edgard Blücher, 1987.

CARRISSO, R. C., CORREIA, J. G. C. Classificação. In: LUZ, A. B. et alii (ed.). Tratamento de minérios ( $3^{\mathrm{a}}$ ed). Rio de Janeiro: CETEM/MCT, 2002.

LYNCH, A. J. Mineral crushing and grinding circuits - their simulation, optimization, design and control. Elsevier, 1977.

LYNCH, A. J., RAO, T. C. Studies in the operating characteristics of hydrocyclones classifiers. Indian Journal of Technology. v. 6, p. 106-114, April 1968.

NAGESWARARAO, K. Reduced efficiency curves of industrial hydrocyclones - an analysis for plant practice. Minerals Engineering. v. 12, n. 5, p. 517-544, 1999.

PLITT, L. R. A Mathematical model of the hydrocyclone classifier. CIM Bulletin. p. 114-123, December 1976.

ZHAO, B. Development of a new method for evaluating cyclone efficiency. Chemical Engineering and Processing. v. 44, p.447451, 2005.

Artigo recebido em 25/02/2003 e aprovado em 25/01/2005. 